九州大学学術情報リポジトリ

Kyushu University Institutional Repository

\title{
Completeness of Depth-Bounded Resolution for Weakly Reducing Programs
}

Arimura, Hiroki

Department of Information Systems, Kyushu University

http://hdl. handle. net/2324/3128

出版情報 : RIFIS Technical Report. 21，1989-12-30. Research Institute of Fundamental Information Science, Kyushu University バージョン:

権利関係 : 


\title{
RIFIS Technical Report
}

\section{Completeness of Depth-Bounded Resolution for Weakly Reducing Programs}

\author{
Hiroki Arimura
}

December 30, 1989

Research Institute of Fundamental Information Science

Kyushu University 33

Fukuoka 812, Japan

E-mail: arim@rifis.sci.kyushu-u.ac.jp Phone:092(641)1101 Ext.2329 


\title{
Completeness of Depth-Bounded Resolution for Weakly Reducing Programs
}

\author{
Hiroki Arimura \\ Department of Information Systems \\ Kyushu University 39, Kasuga 816 \\ arim@rifis1.sci.kyusyu-u.ac.jp
}

\begin{abstract}
SLDNF-resolution procedure is not complete with respect to the perfect model semantics for logic programs in general. In this paper, we introduce two classes of logic programs containing function symbols, reducing programs and weakly reducing programs, which are characterized by the size of atom. For these classes of programs, we prove the completeness of the derivation procedure which makes use of depth-bound. First, we introduce the local finiteness of Herbrand base of a program, and prove the finite fixpoint property for the class of programs which satisfies the local finiteness. Further, we show that the class of weakly reducing programs, a subclass of locally stratified programs, has some syntactic conditions, and prove that it has the finite fixpoint property. Using the finite fixpoint property, we prove the completeness of depth-bounded derivations for weakly reducing programs. In particular, we prove the completeness of unbounded derivations for reducing programs.
\end{abstract}

\section{Introduction}

The completeness of the derivation procedure is an important problem in logic programming. Any Turing machine can be simulated by a definite program[15]. Since the termination problem of Turing machines is undecidable, the completeness of a derivation procedure in logic programming does not hold in general. However, there are some completeness results for restricted classes of logic programs.

SLDNF-resolution is complete for the class of allowed hierarchical programs [10], which can not represent any recursion. QSQR/SLS query evaluation procedure[7] and OLDTNFresolution[14] are complete for the class of allowed stratified databases[1], which is the class of programs containing no function symbols. The class has enough power to express deductive databases. However, many of small efficient logic programs like list-operation programs are not contained in the class, because the function-freeness prevents us from using complex terms representing data structures such as List and Binary-Tree. The allowedness rejects many of "acceptable" programs[5],[11] including the above mentioned small efficient logic 
programs. For example, the program P1 below is neither function-free nor allowed.

$$
\mathrm{P} 1=\left\{\begin{array}{l}
\text { member }(x, \operatorname{cons}(x, z)) \\
\operatorname{member}(x, \operatorname{cons}(y, z)) \leftarrow \operatorname{member}(x, z)
\end{array}\right\}
$$

The stratifiedness is a condition to ensure the freedom from recursive negation. The following program P2 satisfies the freedom and has a clear declarative semantics, that is, even numbers represented by the successor function. However, it is not stratified.

$$
\mathrm{P} 2=\left\{\begin{array}{l}
\operatorname{even}(s(x)) \leftarrow \neg \operatorname{even}(x) \\
\operatorname{even}(0)
\end{array}\right\}
$$

In this paper, we discuss the iterative fixpoint semantics and the procedural semantics for these natural and efficient logic programs, not for deductive databases. More precisely, we discuss three conditions, "local stratified", "locally finite" and "local-variable-free" instead of "stratified", "functions-free" and "allowed", respectively.

First, we extend the notion of the finiteness of Herbrand base to the local finiteness and prove the finite fixpoint property for locally finite stratified programs. Further, we introduce two classes of logic programs, weakly reducing programs and reducing programs, which are characterized by the size of atom. These classes include many of small efficient logic programs. We give some syntactic conditions of two classes and prove that these classes are locally finite stratified and local-variable-free. Moreover, we prove the completeness of depth-bounded derivation procedures for weakly reducing programs using finite fixpoint property for locally finite programs. In particular, we prove the completeness of unbounded derivation procedures for its subclass, reducing programs.

\section{Preliminaries}

A program clause is a clause of the form $A \leftarrow L_{1}, \ldots, L_{n}$. A goal is a clause of the form $\leftarrow L_{1}, \ldots, L_{n}$, where $A$ is an atom and $L_{1}, \ldots, L_{n}$ are literals. A program is a finite nonempty set of program clauses.

A definite program clause is a clause of the form $A \leftarrow A_{1}, \ldots, A_{n}$. A definite goal is a clause of the form $\leftarrow A_{1}, \ldots, A_{n}$, where $A$ and $A_{1}, \ldots, A_{n}$ are atoms. A definite program is a finite non-empty set of definite program clauses.

A ground term is a term containing no variables. Similarly, a ground atom is an atom containing no variables. Let $L$ be a first order language. The Herbrand base $B_{L}$ is the set of all ground atoms. (In case $L$ has no constants, we add some constant "a" to the first order language $L$.) Let $P$ be a program. The Herbrand instantiation of $P$, denoted by $g r o u n d(P)$, is the set of all ground instances of a program $P$. An Herbrand model $M$ is a subset of $B_{P}$ which is a model of $P$. In this paper, we only deal with Herbrand models, so we call an Herbrand model a model. 
Locally stratified programs were introduced by Przymusinski[12], who generalized the class of stratified programs. A program $P$ is locally stratified if there is a partition

$$
B_{P}=H_{0}+H_{1}+\cdots+H_{\alpha}+\cdots(\alpha<\omega)
$$

of an Herbrand base which satisfies the following three conditions for every program clause $A \leftarrow L_{1}, \ldots, L_{n} \in \operatorname{ground}(P)$ and any $i=1, \ldots, n$ :

1. $A \in H_{k}(k \geq 0)$,

2. $D_{i} \in \bigcup\left\{H_{j} \mid j \leq k\right\}$, if $L_{i}$ is positive literal $D_{i}$,

3. $D_{i} \in \bigcup\left\{H_{j} \mid j<k\right\}$, if $L_{i}$ is negative literal $\neg D_{i}$.

We call the partition $H_{0}, \ldots, H_{\alpha}, \ldots$ the local stratification of $B_{P}$, and the partition $P_{0}, \ldots, P_{\alpha}, \ldots$ of $\operatorname{ground}(P)$ the local stratification of $\operatorname{ground}(P)$, where

$$
P_{\alpha}=\left\{A \leftarrow A_{1}, \ldots, A_{q} \in \operatorname{ground}(P) \mid A \in H_{\alpha}, q \geq 0\right\}
$$

and $\alpha<\omega$. The level of a positive literal $A$ is $\alpha$ if $A \in H_{\alpha}$, and the level of a negative literal $\neg A$ is $\alpha+1$ if $A \in H_{\alpha}$. Every stratified program is locally stratified.

In this paper, we consider the perfect model semantics as the semantics for locally stratified programs. The perfect model is a minimal model with respect to a preference order between models. A locally stratified program has a unique perfect model independent of the stratification.

We now define the perfect model. Let $P$ be a program, $A, B, C, D \in B_{P}$. Then, priority orders $\prec$ and $\preceq$ on Herbrand base $B_{P}$ is given as follows:

1. (condition I.) $A \prec B$ if there exists a program clause $A \leftarrow A_{1}, \ldots, \neg B, \ldots, A_{n} \in$ $\operatorname{ground}(P)(n>0)$. (We say this clause defines $A \prec B$.)

2. (condition II.) $A \preceq B$ if there exists a program clause $A \leftarrow A_{1}, \ldots, B, \ldots, A_{n} \in$ $\operatorname{ground}(P)(n>0)$. (We say this clause defines $A \preceq B$.)

3. (transitivity of $\preceq$ ) $A \preceq C$ if $A \preceq B$ and $B \preceq C$.

4. (transitivity of $\prec) A \prec C(D \prec B)$ if $A \preceq B$ and $B \prec C(D \prec A)$.

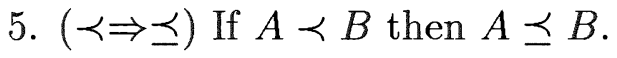

6. (closure axiom) $\prec$ and $\preceq$ are only the relations defined above.

Let $M, N$ be distinct models of $P$. $M$ is preferable to $N$ if for every $A \in M-N$ there exists a $B \in N-M$ such that $A \prec B$. We call a model $M$ a perfect model of $P$ if no other model of $P$ is preferable to $M$. 
The perfect model can be characterized by the fixpoint of a mapping $T_{P}: 2^{B_{P}} \rightarrow 2^{B_{P}}$ for logic programs containing negative literals in the bodies of clauses. We define ordinal powers of the mapping $T_{P}$ by

$$
T_{P}(I)=\left\{\begin{array}{l|l}
A \in B(P) & \begin{array}{l}
\text { for some literals } L_{1} \wedge \ldots \wedge L_{n} \\
A \leftarrow L_{1}, \ldots, L_{n} \in \operatorname{ground}(P) \\
\text { and } M \vDash L_{1} \wedge \ldots \wedge L_{n}
\end{array}
\end{array}\right\}
$$

$$
T_{P} \uparrow 0(I)=I
$$

$T_{P} \uparrow \alpha(I)=T_{P}\left(T_{P} \uparrow(\alpha-1)(I)\right) \cup T_{P} \uparrow(\alpha-1)(I)$, if $\alpha$ is a successor ordinal,

$T_{P} \uparrow \alpha(I)=\bigcup\left\{T_{P} \uparrow \beta(I) \mid \beta<\alpha\right\}$, if $\alpha$ is a limit ordinal.

An ordinal $\gamma_{\alpha}$ is the closure ordinal if $\gamma_{\alpha}$ is a least ordinal such that

$$
T_{P_{\alpha}} \uparrow \gamma_{\alpha}(I)=T_{P_{\alpha}} \uparrow\left(\gamma_{\alpha}+1\right)(I)
$$

For a locally stratified program, the closure ordinal $\gamma_{\alpha} \leq \omega$ for any $\alpha<\omega$. Now we define the fixpoint model of a locally stratified program[4].

Definition 1. Let $P$ be a locally stratified program, $P_{0}, \ldots, P_{\alpha}, \ldots(\alpha<\omega)$ be a local stratification of ground $(P)$. A fixpoint model $M_{P}$ is defined as follows:

$$
\begin{aligned}
M_{0} & =T_{P_{0}} \uparrow \omega(\phi), \\
M_{\alpha} & =T_{P_{\alpha}} \uparrow \omega\left(M_{\alpha-1}\right) \quad(\alpha<\omega), \\
M_{P} & =\bigcup\left\{M_{\alpha} \mid \alpha<\omega\right\} .
\end{aligned}
$$

Note that $\omega$ is the closure ordinal. The following proposition characterizes the model $M_{P}$ of a locally stratified program $P[4]$.

Proposition 1 (Cavedon89). Let $P$ be a locally stratified program. Then $M_{P}$ is the unique perfect model of $P$.

Hereafter we call this fixpoint model $M_{P}$ a perfect model. The perfect model semantics is a kind of closed world assumption (CWA) [13]. In the semantics, an atom is assigned true if it is in the perfect model $M_{P}$ of a program $P$. Otherwise the atom is assigned false.

Let $P$ be a program and $M$ be an Herbrand model of $P$. A derivation procedure $D$ is sound with respect to $M$ if for all $A \in B_{P}$ the following (a) and (b) hold:

(a) If there exists a successful derivation sequence for $P \cup\{\leftarrow A\}$ then $A$ is true in $M$.

(b) If every derivation sequence for $P \cup\{\leftarrow A\}$ is failed then $A$ is false in $M$.

A derivation procedure $D$ is complete with respect to $M$ if for all $A \in B_{P}$ the following (c) and (d) hold: 
(c) If $A$ is true in $M$ then there exists a successful derivation sequence for $P \cup\{\leftarrow A\}$.

(d) If $A$ is false in $M$ then every derivation sequence for $P \cup\{\leftarrow A\}$ is failed.

Note that the derivation sequence may be infinite for logic program in general.

We introduce some notations for our discussions. An expression is either a term, a literal, a conjunction or disjunction of literals. The size of an expression $e$, denoted by $|e|$, is the total number of occurrences of variable symbols, constant symbols, function symbols and predicate symbols in $e$. Note that $|\neg A|=|A|$ for negative literal $\neg A$.

Example 1. Let $x$ and $y$ be variable symbols, $a$ and [] be constant symbols, cons be a function symbol and $p$ be a predicate symbol. Then,

$$
\begin{gathered}
|x|=1, \quad|a|=1, \\
|\operatorname{cons}(a, \operatorname{cons}(x,[]))|=5, \\
|\neg p(x)|=2 .
\end{gathered}
$$

Let $o(x, A)$ be the number of all occurrences of a variable $x$ in an atom $A$, and let $v(A)$ be the set of all variables in atom $A$. Let $\sharp(S)$ be the number of elements in a set $S$, and let

$$
\left.S\right|_{n}=\{A \in S|| A \mid \leq n\}
$$

where $S$ is a set of expressions.

An order $\prec$ is noetherian if there exists no infinite sequence $d_{1}, d_{2}, d_{3}, \ldots$ such that $d_{1} \prec d_{2} \prec d_{3} \prec \ldots$.

\section{Locally Finite Stratified Programs}

In this section, we consider the termination problem in logic programming. If $B_{P}$ is finite then the closure ordinal $\gamma$ of the mapping $T_{P}$ is finite. However, $B_{P}$ may be infinite if a program $P$ contains function symbols. Thus, $\gamma$ may not be infinite. Originally the notion of stratification was introduced for the consistency problem of the completed programs. On the other hand, we will use it for the termination problem of programs. Now we define the notion of the locally finite stratification.

Definition 2. Let $P$ be a locally stratified program and $H_{0}, \ldots, H_{\alpha}, \ldots(\alpha<\omega)$ be the local stratification of $B_{P} . P$ is locally finite stratified if

$$
H^{\alpha}=\bigcup\left\{H_{j} \mid j \leq \alpha\right\}
$$

is a finite set for any $\alpha<\omega$. We call $H_{0}, \ldots, H_{\alpha}, \ldots(\alpha<\omega)$ the locally finite stratification of $B_{P}$, and the corresponding local stratification of ground $(P)$ the locally finite stratification of $\operatorname{ground}(P)$. 
For a definite program $P, B_{P}$ is a local stratification of $B_{P}$. Thus, definite program is locally stratified. However, $B_{P}$ may not be the locally finite stratification for a definite program $P$ if $P$ contains function symbols.

Example 2. The definite program

$$
\mathrm{P} 3=\left\{\begin{array}{l}
p(f(x)) \leftarrow p(x) \\
p(x) \leftarrow p(x) \\
p(a)
\end{array}\right\}
$$

has $B_{\mathrm{P} 3}$ as a trivial local stratification of Herbrand base. However, $B_{\mathrm{P} 3}$ is not a locally finite stratification because $B_{\mathrm{P} 3}=\left\{p\left(f^{i}(a) \mid i<\omega\right\}\right.$ is not finite. On the other hand, there is another local stratification $H_{0}, \ldots, H_{\alpha}, \ldots(\alpha<\omega)$ of $B_{\mathrm{P} 3}$ such that $H_{\alpha}=\left\{p\left(f^{\alpha}(a)\right\}\right.$. Since $H^{\alpha}=\left\{p\left(f^{i}(a) \mid i \leq \alpha\right\}\right.$ is finite for any $\alpha<\omega$, the local stratification $H_{0}, \ldots, H_{\alpha}, \ldots$ is a locally finite stratification. Hence, P3 is a locally finite stratified program, and has countably infinite strata.

Lemma 2. Let $P$ be a locally stratified program, $P_{0}, \ldots, P_{\alpha}, \ldots(\alpha<\omega)$ be the local stratification of ground $(P)$ and $M \subseteq B_{P}$. Then,

$$
T_{P_{\alpha}} \uparrow j(M) \subseteq T_{P_{\alpha}} \uparrow(j+1)(M)
$$

Proof. By the definition of ordinal powers of $T_{P}$, the result immediately holds.

Lemma 3. Let $P$ be a locally stratified program, $P_{0}, \ldots, P_{\alpha}, \ldots(\alpha<\omega)$ be the local stratification of ground $(P)$ and $A \in B_{P}$. Suppose $M_{P_{\alpha}}=T_{P_{\alpha-1}} \uparrow \omega\left(M_{P_{\alpha-1}}\right)$ and $M_{P_{-1}}=\phi$. If there exists a $k<\omega$ such that

$$
T_{P_{\alpha}} \uparrow(k)\left(M_{\alpha-1}\right) \supseteq T_{P_{\alpha}} \uparrow(k+1)\left(M_{\alpha-1}\right),
$$

then for any $j>k$

$$
T_{P_{\alpha}} \uparrow(k)\left(M_{\alpha-1}\right) \supseteq T_{P_{\alpha}} \uparrow(j)\left(M_{\alpha-1}\right)
$$

Proof. It suffices to prove only the case $j=k+2$. First suppose $A \in T_{P_{\alpha}} \uparrow(k+2)\left(M_{\alpha-1}\right)$. Then,

$$
A \in T_{P_{\alpha}}\left(T_{P_{\alpha}} \uparrow(k+1)\left(M_{\alpha-1}\right)\right) \cup T_{P_{\alpha}} \uparrow(k+1)\left(M_{\alpha-1}\right) .
$$

If $A \in T_{P_{\alpha}} \uparrow(k+1)\left(M_{\alpha-1}\right)$ then it immediately holds. Thus, we suppose

$$
A \in T_{P_{\alpha}}\left(T_{P_{\alpha}} \uparrow(k+1)\left(M_{\alpha-1}\right)\right) .
$$

There is a clause $A \leftarrow L_{1}, \ldots, L_{q} \in P_{\alpha}$ such that

$$
T_{P_{\alpha}} \uparrow(k+1)\left(M_{\alpha-1}\right) \models L_{1} \wedge \ldots \wedge L_{q} .
$$

There are two cases to be considered for each $L_{i}$. 
1. If $L_{i}$ is a positive literal $B$ then $B \in T_{P_{\alpha}} \uparrow(k+1)\left(M_{\alpha-1}\right)$. By the assumption of lemma, $B \in T_{P_{\alpha}} \uparrow(k)\left(M_{\alpha-1}\right)$.

2. If $L_{i}$ is a negative literal $\neg B$ then $B \notin T_{P_{\alpha}} \uparrow(k+1)\left(M_{\alpha-1}\right)$. By the definition of ordinal powers of the mapping, $B \notin T_{P_{\alpha}} \uparrow(k)\left(M_{\alpha-1}\right)$.

By the above 1 and $2, T_{P_{\alpha}} \uparrow(k)\left(M_{\alpha-1}\right) \models L_{1} \wedge \ldots \wedge L_{q}$. We obtain $A \in T_{P_{\alpha}} \uparrow(k+1)\left(M_{\alpha-1}\right)$. By the assumption of lemma, $A \in T_{P_{\alpha}} \uparrow(k)\left(M_{\alpha-1}\right)$. Thus,

$$
T_{P_{\alpha}} \uparrow(k)\left(M_{\alpha-1}\right) \supseteq T_{P_{\alpha}} \uparrow(k+2)\left(M_{\alpha-1}\right) .
$$

The remainder of the proof proceeds on in the same way by an induction on $j$.

Definition 3. Let $P$ be a locally stratified program, $H_{0}, \ldots, H_{\alpha}, \ldots(\alpha<\omega)$ be the local stratification of $B_{P}$, and let $M_{P_{\alpha}}=T_{P_{\alpha-1}} \uparrow \omega\left(M_{P_{\alpha-1}}\right)$ and $M_{P_{-1}}=\phi$.

Then, we define a lattice

$$
\Lambda_{\alpha}=\left\{M_{\alpha-1} \cup I \mid I \subseteq H_{\alpha}\right\}
$$

Lemma 4. Let $P$ be a locally finite stratified program, $H_{0}, \ldots, H_{\alpha}, \ldots(\alpha<\omega)$ be the locally finite stratification of $B_{P}$. If

$$
S_{0} \subseteq S_{1} \subseteq \ldots \subseteq S_{i} \subseteq \ldots\left(S_{i} \in \Lambda_{\alpha}, i \geq 0\right)
$$

then there exists a $k \leq \sharp\left(H_{\alpha}\right)$ such that $S_{j}=S_{k}$ for any $j>k$.

Proof. By the definition of $\Lambda_{\alpha}$, we can assume that $S_{i}=M_{\alpha-1} \cup I_{i}$ for some $I_{i} \subseteq H_{\alpha}$ and any $i \geq 0$. Then $I_{0} \subseteq I_{1} \subseteq \ldots \subseteq I_{i} \subseteq \ldots(i \geq 0)$. Since $I_{i} \subseteq H_{\alpha}$ for any $i \geq 0$, there exists a $k \leq \sharp\left(H_{\alpha}\right)$ such that $I_{j}=I_{k}$ for any $j>k$. Then $S_{j}=S_{k}$ for any $j>k$.

Lemma 5. Let $P$ be a locally finite stratified program, $H_{0}, \ldots, H_{\alpha}, \ldots(\alpha<\omega)$ be the locally finite stratification of $B_{P}$. Then, the closure ordinal $\gamma_{\alpha} \leq \sharp\left(H_{\alpha}\right)$ for any $\alpha<\omega$.

Proof. By Lemma 2, for any $j<\omega$

$$
T_{P_{\alpha}} \uparrow 0\left(M_{\alpha-1}\right) \subseteq \ldots \subseteq T_{P_{\alpha}} \uparrow j\left(M_{\alpha-1}\right) \subseteq T_{P_{\alpha}} \uparrow j+1\left(M_{\alpha-1}\right) \subseteq \ldots \subseteq T_{P_{\alpha}} \uparrow \omega\left(M_{\alpha-1}\right)
$$

By definitions of the local stratification of $\operatorname{ground}(P)$ and ordinal powers of $T_{P}, T_{P_{\alpha}} \uparrow$ $j\left(M_{\alpha-1}\right) \in \Lambda_{\alpha}$ for any $j \leq \omega$. By Lemma 3 and Lemma 4 , there exists a $k \leq \sharp\left(H_{\alpha}\right)$ such that $T_{P_{\alpha}} \uparrow k\left(M_{\alpha-1}\right)$ is a fixpoint $T_{P_{\alpha}} \uparrow \omega\left(M_{\alpha-1}\right)$.

The closure ordinal $\gamma_{\alpha}$ corresponding to each $H_{\alpha}$ is finite for a locally finite stratified program. Now we show the finite fixpoint property for locally finite stratified programs. 
Theorem 6. Let $P$ be a locally finite stratified program, $H_{0}, \ldots, H_{\alpha}, \ldots(\alpha<\omega)$ be the locally finite stratification of $B_{P}$ and $P_{0}, \ldots, P_{\alpha}, \ldots(\alpha<\omega)$ be the locally finite stratification of ground $(P)$. If $\gamma_{\alpha}$ is the closure ordinal of a mapping $T_{P_{\alpha}}: \Lambda_{\alpha} \rightarrow \Lambda_{\alpha}$ for any $\alpha<\omega$, then

$$
\gamma_{0}+\ldots+\gamma_{\alpha} \leq \sharp\left(H^{\alpha}\right)
$$

Proof. By Lemma 5, if $T_{P_{\alpha}} \uparrow \omega\left(M_{\alpha-1}\right)=T_{P_{\alpha}} \uparrow \gamma_{\alpha}\left(M_{\alpha-1}\right)$ for any $\alpha<\omega$ then $\gamma_{\alpha} \leq \sharp\left(H_{\alpha}\right)$. Hence,

$$
\begin{aligned}
\gamma_{0}+\ldots+\gamma_{\alpha} & \leq \sharp\left(H_{0}\right)+\ldots+\sharp\left(H_{\alpha}\right) \\
& =\sharp\left(H_{0} \cup \ldots \cup H_{\alpha}\right) \\
& =\sharp\left(H^{\alpha}\right) .
\end{aligned}
$$

The above corollary asserts that we can decide whether a ground atom in $H_{\alpha}$ is true or false on the perfect model of a program $P$ by applying operators $T_{P_{j}}(j=0, \ldots, \alpha)$ at most $\sharp\left(H^{\alpha}\right)$ times. The following corollary is an extension of the theorem in Yamamoto[17] for elementary formal systems[2].

Corollary 7. Let $P$ be a definite program, and let $P$ be locally finite stratified, $H_{0}, \ldots, H_{\alpha}, \ldots(\alpha<$ $\omega)$ be the locally finite stratification of $B_{P}$ and $A \in B_{P}$. If $A \in H_{\alpha}$ then

$$
A \in T \uparrow \omega(\phi) \Longleftrightarrow A \in T \uparrow n(\phi)
$$

for the finite ordinal $n=\sharp\left(H^{\alpha}\right)$.

Proof. We consider the increasing sequence

$$
T_{P_{0}} \uparrow 0(\phi), \ldots, T_{P_{0}} \uparrow \gamma_{0}(\phi), \ldots, T_{P_{\alpha}} \uparrow \gamma_{\alpha}\left(M_{\alpha-1}\right)
$$

Since a definite program has no negative literals in the body of a clause, we can consider the lattice $2^{B_{P}}$ whose least element is $\phi$, instead of the sequence $\Lambda_{0}, \ldots, \Lambda_{\alpha}$ of lattices. Thus, the increasing sequence above is reduced to the sequence

$$
T_{P} \uparrow 0(\phi), \ldots, T_{P} \uparrow \gamma_{0}(\phi), \ldots, T_{P} \uparrow\left(\gamma_{0}+\ldots+\gamma_{\alpha}\right)(\phi)
$$

Hence, the result holds by Theorem 6 .

\section{Depth-Bounded BFNF-Derivation}

We introduce the notion of depth-bounded BFNF-derivation to discuss the termination property of logic programs more precisely. "BF" stands for "Breadth First", and "NF" 
stands for "Negation as Failure". A BFNF-derivation is a variant of the usual SLDNFresolution, where in each goal all literals are selected. This computation rule ensures that every derivation is a fair[9] derivation.

We define a $\mathrm{BFNF} / d$-derivation for programs, which is an extension of the $(P, E)$ derivation[6]. The $\mathrm{BFNF} / d$-derivation sequence is a BFNF-derivation sequence which is bounded by the depth-bound $d$, where $d$ is an ordinal. Throughout this paper, we assume $d \leq \omega$.

Definition 4. Let $G$ be a goal $\leftarrow L_{1}, \ldots, L_{n}\left(L_{i}\right.$ is a literal) and $P$ be a program. If there are a substitution $\theta$ and an $n$-tuple $\left(C_{1}, \ldots, C_{n}\right)$ (each $C_{i}$ is a literal) such that the following conditions 1,2 and 3 hold for every $i=1, \ldots, n$, then a goal $\leftarrow R_{1}, \ldots, R_{n}$ is derived from $G$ and $P$ using the most general unifier (mgu) $\theta$.

1. $\theta$ is an mgu of conjunctions of literals $\left(L_{1}, \ldots, L_{n}\right)$ and $\left(C_{1}, \ldots, C_{n}\right)$.

2. If $L_{i}$ is a positive literal $A$ then

(a) $C_{i}$ is a positive literal $B$ and there is a clause $B \leftarrow M_{1}, \ldots, M_{q}(q \geq 0)$ which is a variant of a clause in $P$. (We call each $M_{j}(j=1, \ldots, q)$ a child of $L_{i}$.)

(b) $R_{i}$ is a conjunction of literals $\left(M_{1}, \ldots, M_{q}\right) \theta$.

3. If $L_{i}$ is a ground negative literal $\neg A$ then

(a) $C_{i}$ is $\neg A$ and every $\mathrm{BFNF} / d$-derivation sequence for $P \cup\{\leftarrow A\}$ is failed. (If a literal $M$ is a child of $A$ in the BFNF/d-derivation sequence for $P \cup\{\leftarrow A\}$ then we call $M$ the child of $L_{i}$. Moreover, we call derivation sequences for $P \cup\{\leftarrow A\}$ partial derivation sequences for $P \cup\{\leftarrow \neg A\}$.)

(b) $R_{i}$ is empty.

Definition 5. Let $P$ be a program, $G$ be a goal and $d \leq \omega$ be a depth-bound. A $B F N F / d$ derivation sequence for $P \cup\{G\}$ consists of a sequence $G_{0}=G, G_{1}, \ldots, G_{\alpha}, \ldots$ of goals and a sequence $\theta_{1}, \theta_{2}, \ldots, \theta_{\alpha}, \ldots(\alpha \leq d)$ of mgus such that each $G_{i+1}$ is derived from $G_{i}$ and $P$ using $\theta_{i+1}$.

A derivation sequence $G_{0}, G_{1}, \ldots$ is successful if there is an $n$ such that $G_{n}$ is an empty clause. A derivation sequence $G_{0}, G_{1}, \ldots$ is floundered if there is an $n$ such that $G_{n}$ contains non-ground negative literals. A derivation sequence $G_{0}, G_{1}, \ldots, G_{n}$ is failed with length $n$ if either any goal cannot be derived from $G_{n}$ which contains no non-ground negative literal (in this case the sequence is finitely failed), or $n=d$ and a goal $G_{n}$ is not empty (in this case the sequence is depth-bound-failed). Otherwise a derivation sequence $G_{0}, G_{1}, \ldots$ has infinite length.

A BFNF/d-derivation is successful if there exists a successful $\mathrm{BFNF} / d$-derivation sequence for $P \cup\{G\}$. A BFNF/d-derivation is failed if there exists an $n$ such that every $\mathrm{BFNF} / d$-derivation sequence for $P \cup\{G\}$ is failed within length $n$. 
We define a BFNF/d-tree for measuring only the time derivation procedure requires.

Definition 6. Let $P$ be a program, $G$ be a goal and $D$ be a BFNF/d-derivation sequence $G_{0}, G_{1}, \ldots$ for $P \cup\{G\}$. A $B F N F / d$-tree $T$ for $D$ is a tree defined as follows:

1. a root of $T$ is a literal in $G$,

2. every node in $T$ is a literal in $D$ or in partial derivation sequences of $D$,

3. if a literal $B$ is a child of a literal $A$ in $D$ or in a partial derivation sequence of $D$ then $B$ is a child of $A$ in $T$.

The depth of a BFNF/d-tree $T$ is the maximum length of branches in $T$.

We define an unbounded BFNF-derivation and a depth-bounded BFNF-derivation. If a depth-bound $d$ is the smallest limit ordinal $\omega$ then we call the derivation sequence an unbounded BFNF-derivation sequence. If $d<\omega$ then we call the derivation sequence a depth-bounded BFNF-derivation sequence with depth-bound $d$. We define successful, floundered, failed, infinite derivation sequences and BFNF-trees in the same way as above. Note that a failed unbounded BFNF-derivation sequence is a finitely failed unbounded BFNFderivation sequence. Because an unbounded BFNF-derivation has no derivation sequence which is depth-bound-failed. Note also that a depth-bounded BFNF-derivation has no infinite derivation sequence.

An unbounded BFNF-derivation is sound like the usual SLDNF-resolution. We show the soundness of a successful unbounded BFNF-derivation and a finitely failed unbounded BFNF-derivation.

Proposition 8. Let $P$ be a local-variable-free program, $M_{P}$ be the perfect model of $P$, and $L$ be a ground literal. Then the following (a) and (b) hold:

(a) If there exists an unbounded BFNF-derivation for $P \cup\{\leftarrow L\}$ then $M_{P} \models L$.

(b) If there exists an $n$ such that every unbounded BFNF-derivation for $P \cup\{\leftarrow L\}$ is finitely failed within the length $n$ then $M_{P} \models \neg L$.

Corollary 9. An unbounded BFNF-derivation for a local-variable-free program is sound with respect to its perfect model semantics.

A depth-bounded BFNF-tree has no infinite branch, since a depth-bound $d$ is less than $\omega$.

Lemma 10. Let $P$ be a locally stratified program, $L$ be a literal, and $d$ be a depth-bound. If the level of $L$ is $\alpha$ and a depth-bound $d<\omega$ then a depth-bounded BFNF-tree for $P \cup\{G\}$ is a finite tree whose depth is at most $(\alpha+1) \cdot d$. 
Proof. The length of each depth-bounded BFNF-derivation sequences and partial derivation sequences with depth-bound $d$ is at most $d$. On the other hand, the number of strata whose level is lower than or equal to $\alpha$ is at most $\alpha+1$. Hence, the length of every branch of the depth-bounded BFNF-tree is less than or equal to $(\alpha+1) \cdot d$.

\section{Weakly Reducing Programs and Reducing Programs}

Definition 7. A program clause $A \leftarrow L_{1}, \ldots, L_{n}$ is weakly reducing if for any substitution $\theta$ and any $i=1, \ldots, n$ the following conditions 1 and 2 hold:

1. $|A \theta| \geq\left|L_{i} \theta\right|$ if $L_{i}$ is a positive literal.

2. $|A \theta|>\left|L_{i} \theta\right|$ if $L_{i}$ is a negative literal.

A program clause $A \leftarrow L_{1}, \ldots, L_{n}$ is reducing if

$$
|A \theta|>\left|L_{i} \theta\right|
$$

for any substitution $\theta$ and any $i=1, \ldots, n$.

A program $P$ is weakly reducing (reducing) if every program clause in $P$ is weakly reducing (reducing).

Example 3. A program P1 and a program P2 shown in Section 1 are reducing programs. Every reducing programs are also weakly reducing programs.

The proposition below provides some syntactic conditions that programs be weakly reducing and reducing.

Proposition 11. Let $P$ be a program. Suppose $P$ contains at least one function symbol. $A$ clause $A \leftarrow L_{1}, \ldots, L_{n}$ in $P$ is weakly reducing (reducing) if and only if the following conditions (a),(b) and (c) hold for any variable $x$ in the clause and any $i=1, \ldots, n$ :

(a) $o(x, A) \geq o\left(x, L_{i}\right)$.

(b) $|A| \geq(>)\left|L_{i}\right|$, if $L_{i}$ is a positive literal.

(c) $|A|>\left|L_{i}\right|$, if $L_{i}$ is a negative literal.

Suppose $P$ contains no function symbols. Then a clause $A \leftarrow L_{1}, \ldots, L_{n}$ in $P$ is weakly reducing (reducing) if and only if above conditions (b) and (c) hold for any variable $x$ in the clause and any $i=1, \ldots, n$. 
Proof. Let $C$ be a program clause $A \leftarrow L_{1}, \ldots L_{m}(n \geq 0), v(C)$ be a set of variables $\left\{x_{1}, \ldots x_{q}\right\}$ and $\theta$ be a substitution. We assume that $\theta$ is a substitution restricted to variables in $C$ without loss of generality. Let $\theta=\left\{x_{j}:=t_{j} \mid j=1, \ldots, q\right\}$. Then, for $i=1, \ldots, n$

$$
|A \theta|-\left|L_{i} \theta\right|=\sum_{j=1}^{q}\left(o\left(x_{j}, A\right)-o\left(x_{j}, L_{i}\right)\right)\left(\left|t_{j}\right|-\left|x_{j}\right|\right)+|A|-\left|L_{i}\right| .
$$

$(\Rightarrow$ part) Suppose $P$ contains function symbols, and conditions (a),(b) and (c) hold for any $i=1, \ldots, n$. Since $\left|t_{j}\right|-\left|x_{j}\right| \geq 0$, the following conditions (d) and (e) hold for any $\theta$ :

(d) $|A \theta| \geq\left|L_{i} \theta\right|$, if $L_{i}$ is a positive literal.

(e) $|A \theta|>\left|L_{i} \theta\right|$, if $L_{i}$ is a negative literal.

Thus, $C$ is weakly reducing. If there exists no function symbols then (b) and (c) imply (d) and (e). Thus, $C$ is weakly reducing.

( $\Leftarrow$ part) Suppose $C$ is weakly reducing. We show that if there is an $i(1 \leq i \leq m)$ such that one of the conditions (a),(b) and (c) does not hold then contradiction is derived. Suppose first there is an $i(1 \leq i \leq m)$ such that neither (b) nor (c) holds. Then contradiction is immediately derived for an identity substitution $\theta$. Therefore, conditions (b) and (c) hold for any $i=1, \ldots, n$. This is independent of existence of function symbols.

Suppose next there are $k$ and $m(1 \leq k \leq q, 1 \leq m \leq n)$ such that $o\left(x_{k}, A\right)<o\left(x_{k}, L_{m}\right)$. We take a substitution $\sigma=\left\{x_{j}:=t_{j} \mid j=1, \ldots, q\right\}$ such that

(f) if $j \neq k$ then $t_{j}$ is either a constant symbol or a variable symbol,

(g) if $j=k$ then $t_{k}$ is a term that satisfies

$$
\left|t_{k}\right|>\left\lceil\frac{|A|-\left|L_{m}\right|}{o\left(x_{k}, L_{m}\right)-o\left(x_{k}, A\right)}\right\rceil .
$$

Since $P$ contains at least one function symbol, there exists such a substitution $\sigma$. Hence, $|A \sigma|<\left|L_{m} \sigma\right|$ for the substitution $\sigma$. This contradicts the assumption. Thus, the condition (a) holds for any $i=1, \ldots, n$.

Example 4. Every propositional program not containing negation is weakly reducing. A program

$$
\mathrm{P} 4=\mathrm{P} 2 \cup\{\operatorname{even}(x) \leftarrow \operatorname{even}(x)\}
$$

is weakly reducing. $\mathrm{P} 4$ has the same perfect model semantics as that of $\mathrm{P} 2$. $\mathrm{P} 4 \cup\{\leftarrow$ even $\left.\left(s^{i}(0)\right)\right\}$ has no finite SLDNF-tree, while every SLDNF-tree for P2 and a ground goal is finite.

Throughout this paper, we only consider weakly reducing programs and reducing programs containing function symbols. 


\section{Freedom from Recursive Negation for Weakly Reducing Programs}

The local stratifiedness ensures the freedom from recursive negation. Przymusinski[12] gave a condition:

Proposition 12 (Przymusinski88). A program $P$ is locally stratified if and only if the priority relation $\prec$ on $B_{P}$ is noetherian.

The class of locally stratified programs is undecidable[4]. However, for weakly reducing programs the priority relation $\prec$ on $B_{P}$ is reduced to the partial order on ground atoms with respect to their sizes. Hence, we immediately obtain the following theorem.

Theorem 13. Every weakly reducing program is locally stratified.

Proof. Let $P$ be a weakly reducing program, $A, B, C, D \in B_{P}$. It suffices to prove that the priority relation $\prec$ on $B_{P}$ is noetherian. If $A \prec B$ then the following 1,2 or 3 hold by the definition of $\prec$ :

1. There exists a clause which defines $A \prec B$.

2. There exist $C$ such that $A \prec C \preceq B$ and a clause in $\operatorname{ground}(P)$ which defines $A \prec C$.

3. There exist $D$ such that $A \preceq D \prec B$ and a clause in $\operatorname{ground}(P)$ which defines $D \prec B$. We consider the case 1 . Let $A \leftarrow A_{1}, \ldots, \neg B, \ldots, A_{n}(n>0)$ be a clause to define $A \prec B$. Then $|A|>|B|$ by the definition of weakly reducing programs. In cases 2 and 3 , we also prove that if $A \prec B$ then $|A|>|B|$ in the same way. Thus, the relation $\prec$ can be reduced to the relation $>$. Therefore an increasing sequence with respect to $\prec$ is reduced to a decreasing sequence of natural numbers. Since $>$ is noetherian, so is $\prec$.

Combining the result in Przymusinski[12] and the theorem, we can conclude that a weakly reducing program has a unique perfect model.

\section{Safeness for Negation of Weakly Reducing Programs}

A variable in a program clause is local if it appears only in the body of the clause. The condition "local-variable-free" is an alternative to the condition "allowed" with respect to occurrences of variables. Goals in a derivation sequence turn ground in a top-down manner for local-variable-free programs, while goals in a derivation sequence turn ground in a bottom-up manner for allowed programs.

Definition 8. A program clause $A \leftarrow L_{1}, \ldots, L_{n}$ is local-variable-free if

$$
v(A) \supseteq v(L i)(i=1, \ldots, n) .
$$

A program $P$ is local-variable-free if every program clause in $P$ is local-variable-free. 
Example 5. The program P1 is a local-variable-free program.

Local-variable-free programs are also acceptable programs[5],[11].

Definition 9. Let $P$ be a program, $G$ be a goal and $d$ be an ordinal. $P \cup\{G\}$ is safe for negation if any BFNF/d-derivation and any partial derivation for $P \cup\{G\}$ do not flounder.

We give a condition under which $P \cup\{G\}$ is safe for negation. First we give a lemma for local-variable-free programs.

Lemma 14. Let $P$ be a local-variable-free program and $G$ be a ground goal. Then, every goal in a BFNF/d-tree for $P \cup\{G\}$ is ground.

Proof. Since $P$ is a local-variable-free program, a goal $G_{i+1}$ which is derived from a ground goal $G_{i}$ is ground. Thus, the result holds.

Hence, the next lemma follows immediately from Lemma 14.

Lemma 15. Let $P$ be a local-variable-free program and $G$ be a ground goal. Then $P \cup\{G\}$ is safe for negation.

By the condition (a) in Proposition 11, a weakly reducing program containing function symbols is local-variable-free. Thus, we have:

Lemma 16. Let $P$ be a weakly reducing program contains function symbols and $G$ be $a$ ground goal. Then $P \cup\{G\}$ is safe for negation.

\section{Termination Properties of Reducing Programs}

In Section 6 and Section 7, we have shown that both a weakly reducing program and a reducing program are locally stratified and safe for negation. In this section, we show that the termination property of unbounded BFNF-derivations for reducing programs, which is a subclass of weakly reducing program. First we have:

Lemma 17. Let $P$ be a program. Then, a partial order $|A|>|B|\left(A, B \in B_{P}\right)$ is noetherian, and the length of the decreasing sequence $\left|A_{1}\right|>\left|A_{2}\right|>\cdots$ is at most $\left|A_{1}\right|$.

Lemma 18. Let $P$ be a reducing program containing function symbols and $L$ be a ground literal. Then, every BFNF/d-tree for $P \cup\{\leftarrow L\}$ is finite, and the depth of that tree is at most $|L|$.

Proof. Let $L$ be ground. Since a reducing program containing function symbols is localvariable-free, every goal in a BFNF/d-tree for $P \cup\{\leftarrow L\}$ is a ground goal. We consider a branch in the BFNF/d-tree. Since this branch is a decreasing sequence with respect to $>$, the length of it is at most $|L|$ by Lemma 17. 
By Lemma 15 and Lemma 18, for a reducing program and a ground goal there is neither an unbounded BFNF-derivation which flounders nor an unbounded BFNF-derivation which has infinite length. By those lemmas and Proposition 9, we can prove the following theorem:

Theorem 19. Let $P$ be a reducing program containing function symbols, $M_{P}$ be the perfect model of $P$ and $L$ be a ground literal. Then, the following (a) and (b) hold:

(a) $M_{P} \models L \Longleftrightarrow$

there is a successful unbounded BFNF-derivation for $P \cup\{\leftarrow L\}$.

(b) $M_{P} \vDash \neg L \Longleftrightarrow$

there is an $n$ such that every unbounded BFNF-derivation for $P \cup\{\leftarrow L\}$ is finitely failed.

Proof. By Proposition 8 in Section 4, both a successful unbounded BFNF-derivation sequence and a finitely failed unbounded BFNF-derivation sequence are sound. Hence, we only show " $\Rightarrow$ " part.

(a) Let $L$ be a ground literal. Since a reducing program is weakly reducing, $P \cup\{\leftarrow L\}$ is safe for negation. On the other hand, every unbounded BFNF-derivation sequence for a reducing program and a ground goal has a finite length by Lemma 18. Thus, every unbounded BFNF-derivation sequence for $P \cup\{\leftarrow L\}$ is either successful or finitely failed. Let $M_{P} \models L$. Then, there is no $n$ such that every unbounded BFNF-derivation sequence for $P \cup$ $\{\leftarrow L\}$ is finitely failed within length $n$ by the soundness of finitely failed unbounded BFNFderivation sequence. Thus, there is a successful unbounded BFNF-derivation sequence for $P \cup\{\leftarrow L\}$. Hence, part (a) holds.

(b) Combining the soundness of successful unbounded BFNF-derivation sequences and the finiteness of unbounded BFNF-derivation sequences, part (b) can be proved in the same way as in the above.

Corollary 20. Let $P$ be a reducing program containing function symbols, and $L$ be a ground literal. Then, an unbounded BFNF-derivation for $P \cup\{\leftarrow L\}$ is complete with respect to the perfect model semantics.

Now we consider the termination property of the usual SLDNF-resolution, instead of the BFNF-derivation. Cavedon[4] introduced the notion of locally $\omega$-hierarchical programs, which is an extension of hierarchical programs. He showed that every fair SLDNF-resolution for locally $\omega$-hierarchical programs terminates, and that if moreover a program and a goal are allowed then the SLDNF-resolution is complete with respect to the perfect model semantics. In fact, our reducing programs are locally $\omega$-hierarchical, so we can also obtain the termination property of the SLDNF-resolution for reducing programs in the same way as in Cavedon[4]. 


\section{Termination Properties of Weakly Reducing Programs}

In this section, we give the solution of the termination problem of weakly reducing programs containing function symbols, and prove the correctness of depth-bounded derivations for the class.

Since a weakly reducing program is safe for negation and a depth-bounded BFNF-tree for weakly reducing programs is finite, a depth-bounded BFNF-derivation procedure returns either success or failure in finite time. Though the depth-bounded derivation may not be sound for an arbitrary depth-bound $d$, we can show that for a given program and a ground goal, there is a computable bounded-depth $d$ such that the depth-bounded BFNF-derivation is complete. First we show that the subset of $B_{P}$ bounded by the term size is finite.

Lemma 21. Let $L$ be a first order language, $\Sigma$ be the set of all constant symbols and all function symbols, and $\Pi$ be the set of all predicate symbols in $L$. If both $\Sigma$ and $\Pi$ are finite then $\left.B_{L}\right|_{n}$ is finite and there exists a computable function $f(n)$ such that $\sharp\left(\left.B_{L}\right|_{n}\right) \leq f(n)$ for any $n>0$.

Proof. Let $S(n)$ be the number of all ordered trees each of which has $n$ nodes. Knuth[8] showed the following:

$$
\begin{aligned}
S(n) & =\frac{1}{n}\left(\begin{array}{c}
2(n-1) \\
n-1
\end{array}\right) \\
& =O\left(4^{n} \cdot n^{-\frac{3}{2}}\right) .
\end{aligned}
$$

Now we regard a ground atom as an ordered tree whose node is labeled with the element of $(\Pi \cup \Sigma)$. Since the total number of assignments of elements of $(\Pi \cup \Sigma)$ to labels in an $n$-node tree is $\sharp(\Pi \cup \Sigma)^{S(n)}$, the total number of atoms of size $\leq n$ is less than or equal to

$$
\sum_{k=1}^{n} \sharp(\Pi \cup \Sigma)^{S(k)} .
$$

Therefore,

$$
\sharp\left(\left.B_{L}\right|_{n}\right)=O\left(n \cdot \sharp(\Pi \cup \Sigma)^{4^{n} \cdot n^{-\frac{3}{2}}}\right) .
$$

Thus, we show the local finiteness of weakly reducing programs.

Theorem 22. Every weakly reducing program is locally finite stratified.

Proof. By Theorem 13, a weakly reducing program $P$ is locally stratified. Let

$$
H_{\alpha}=\left\{A \in B_{P}|| A \mid=\alpha+1\right\}(\alpha<\omega) .
$$


Then, $H_{0}, \ldots, H_{\alpha}, \ldots(\alpha<\omega)$ is the local stratification of $B_{P}$. By Lemma 21,

$$
\begin{aligned}
H^{\alpha} & =\bigcup\left\{H_{j} \mid j \leq \alpha\right\} \\
& =\left\{A \in B_{P}|| A \mid \leq \alpha+1\right\} \\
& =\left.B_{P}\right|_{\alpha+1}
\end{aligned}
$$

is finite. Then $P$ is locally finite stratified.

Since the perfect model of a locally stratified program is independent of its stratification, we only consider the local stratification used in the above proof. Now we define the term-size stratification.

Definition 10. Let $P$ be a weakly reducing program. We call the local stratification $H_{0}, \ldots, H_{\alpha}, \ldots(\alpha<\omega)$ of $B_{P}$ such that

$$
H_{\alpha}=\left\{A \in B_{P}|| A \mid=\alpha+1\right\}
$$

the term-size stratification of $B_{P}$. Further we call $P_{0}, \ldots, P_{\alpha}, \ldots(\alpha<\omega)$ corresponding to this stratification the term-size stratification of $\operatorname{ground}(P)$.

We have a property of depth-bounded BFNF-derivation procedures.

Lemma 23. Let $P$ be a weakly reducing program containing function symbols, $L$ be a ground literal, and $d<\omega$ be a depth-bound. Then a depth-bounded BFNF-derivation sequence is either successful or failed.

Proof. This follows immediately from Lemma 10 and Lemma 16.

Though a depth-bounded BFNF-derivation is neither sound nor complete for an arbitrary depth-bound $d$, we can show that there exists a depth-bound $d$ such that a depth-bounded BFNF-derivation is sound and complete with respect to the perfect model of the program.

Theorem 24. Let $P$ be a weakly reducing program containing function symbols, $P_{0}, \ldots, P_{\alpha}, \ldots$ $(\alpha<\omega)$ be the term-size stratification of ground $(P), \gamma_{\alpha}$ be the closure ordinal of $T_{P_{\alpha}}, A \in B_{P}$, and $d$ be a depth-bound. If $A$ is the level $k<\omega$ and $d \geq \sharp\left(\left.B_{P}\right|_{|A|}\right)$, then the following (a),(b) and (c) hold:

(a) There is a successful depth-bounded BFNF-derivation sequence of length $n$ for $P \cup\{\leftarrow A\} \Longrightarrow$ $A \in T_{P_{k}} \uparrow n\left(M_{k-1}\right)$.

(b) $A \in T_{P_{k}} \uparrow n\left(M_{k-1}\right)$ and $\gamma_{0}+\ldots+\gamma_{k-1}+n \leq d \Longrightarrow$

there is a successful depth-bounded BFNF-derivation sequence of length $\leq \gamma_{0}+\ldots+\gamma_{k-1}+n \leq d$ for $P \cup\{\leftarrow A\}$. 
(c) $A \in T_{P_{k}} \uparrow n\left(M_{k-1}\right)$ and $d<\gamma_{0}+\ldots+\gamma_{k-1}+n \Longrightarrow$

there is a successful depth-bounded BFNF-derivation sequence of length $\leq d$ for $P \cup\{\leftarrow A\}$.

Proof. Parts (a),(b) and (c) are proved simultaneously by an induction on the level $k$ of $A$.

(Base step) Suppose the level of $A$ is 0 . Then, $P_{0}$ is a definite program. Restricting the result of Jaffer et al.[6] to the case where $E$ is an equality relation on ordinary first order terms and $P$ is a definite program, we can directly obtain the following (a') and (b'),

(a') there is a successful $(P, E)$-derivation sequence of length $n$ for $P \cup\{\leftarrow A\}$

$\Longrightarrow A \in T_{P} \uparrow n(\phi)$,

(b') $n \leq d$ and $A \in T_{P} \uparrow n(\phi)$

$\Longrightarrow$ there is a successful $(P, E)$-derivation sequence of length $\leq n$ for $P \cup\{\leftarrow A\}$,

where $P$ is a definite program. For a definite program $P$, an unbounded BFNF-derivation coincides with a $(P, E)$-derivation $[6]$ in case $E$ is an equality relation on ordinary first order terms. Furthermore, a successful depth-bounded BFNF-derivation sequence of length $n \leq d$ is a successful unbounded BFNF-derivation sequence. Hence, parts (a) and (b) immediately follows from the soundness (a') and the completeness (b') of a successful $(P, E)$-derivation sequence above.

Now we consider part (c). Suppose $n>d$ and $A \in T_{P_{0}} \uparrow n(\phi)$. Since $P$ is a weakly reducing program, $P_{0}$ is locally finite stratified. Thus, $A \in T_{P_{0}} \uparrow d(\phi)$ by Corollary 7 . Hence, part (c) follows from part (b).

(Induction step) Suppose each part of the theorem holds for any atom of level $\leq k$, and the level of $A$ is $k+1$. Combining parts (b) and (c) of this hypothesis, for any atom $A^{\prime}$ of level $\leq k$ and any $n<\omega$ if $A^{\prime} \in T_{P_{k}} \uparrow n\left(M_{k-1}\right)$ then there is successful depth-bounded BFNF-derivation sequence of length $\leq d$ for $P \cup\left\{\leftarrow A^{\prime}\right\}$. Thus, the following (d) holds for any atom $A$ of level $\leq k$.

(d) Every depth-bounded BFNF-derivation sequence for $P \cup\{\leftarrow A\}$ is

failed with depth-bound $d \geq \sharp\left(\left.B_{P}\right|_{|A|}\right) \Longrightarrow$

$A \notin T_{P_{k}} \uparrow \gamma_{k}\left(M_{k-1}\right)$.

(part (a)) We assume that there is a successful BFNF/d derivation sequence of length $n \leq d$ for $P \cup\{\leftarrow A\}$.

Suppose first $n=1$. Then there exists a clause $A \leftarrow \neg B_{1}, \ldots, \neg B_{q} \in \operatorname{ground}(P)(q \geq 0)$ such that every depth-bounded BFNF-derivation sequence for $P \cup\left\{\leftarrow B_{i}\right\}$ is failed for any $i=1, \ldots, q$. Since $P$ is weakly reducing, $B_{i}$ is level $\leq k$ and $d \geq \sharp\left(\left.B_{P}\right|_{|A|}\right) \geq \sharp\left(\left.B_{P}\right|_{\left|B_{i}\right|}\right)$. By part (d) of main induction hypothesis,

$$
B_{i} \notin T_{P_{k}} \uparrow \gamma_{k}\left(M_{k-1}\right) .
$$


As $\gamma_{k}$ is the closure ordinal of $T_{P_{k}}, T_{P_{k}} \uparrow \gamma_{k}\left(M_{k-1}\right)=M_{k}$. Therefore $M_{k} \models \neg B_{1} \wedge \ldots \wedge \neg B_{q}$. Hence, $T_{P_{k+1}} \uparrow 1\left(M_{k}\right)$.

Suppose next $n>1$, and the result holds for all successful depth-bounded BFNFderivation sequences of length $\leq n-1$. Suppose $G_{0}=\leftarrow A, G_{1}, \ldots, G_{n}$ is the successful depth-bounded BFNF-derivation sequence for $P \cup\{\leftarrow A\}$ of length $n$. Since $P$ is localvariable-free, the derivation sequence $G_{0}, \ldots, G_{n}$ consists of ground goals. Let a ground goal $G_{1}$ be $\leftarrow L_{1}, \ldots, L_{q}$. By the definition of derivation sequences, $G_{1}, \ldots, G_{n}$ is the successful depth-bounded BFNF-derivation sequence of length $n-1 \leq d$ for $P \cup\left\{\leftarrow L_{1}, \ldots, L_{q}\right\}$, and there is a clause $A \leftarrow L_{1}, \ldots, L_{q} \in \operatorname{ground}(P)$. Since $G_{1}, \ldots, G_{n}$ is ground and $P$ is local-variable-free, we can construct a successful depth-bounded BFNF-derivation sequence of length $\leq n-1$ for $P \cup\left\{\leftarrow L_{i}\right\}(i=1, \ldots, q)$. There are three possibilities for each $L_{i}$.

1. If $L_{i}$ is a positive literal $B_{i} \in H_{j}(j \leq k)$ then there is a successful depth-bounded BFNF-derivation sequence of length $n-1$ for $P \cup\left\{\leftarrow B_{i}\right\}$. By part (a) of the main induction hypothesis, $B_{i} \in T_{P_{k}} \uparrow(n-1)\left(M_{k-1}\right)$. Since $P$ is locally stratified, $B_{i} \in$ $T_{P_{k+1}} \uparrow(n-1)\left(M_{k}\right)$.

2. If $L_{i}$ is a positive literal $B_{i} \in H_{k+1}$ then there is a successful depth-bounded BFNFderivation sequence of length $n-1$ for $P \cup\left\{\leftarrow B_{i}\right\}$. By part (a) of the secondary induction hypothesis, $B_{i} \in T_{P_{k+1}} \uparrow(n-1)\left(M_{k}\right)$.

3. If $L_{i}$ is a negative literal $\neg B_{i}\left(B_{i} \in B_{P}\right)$, then $B_{i}$ is level $\leq k$ and every depthbounded BFNF-derivation sequence for $P \cup\left\{\leftarrow B_{i}\right\}$ is failed. Since $P$ is weakly reducing, $d \geq \sharp\left(\left.B_{P}\right|_{|A|}\right) \geq \sharp\left(\left.B_{P}\right|_{\left|B_{i}\right|}\right)$. Thus, $B_{i} \notin T_{P_{k}} \uparrow \gamma_{k}\left(M_{k-1}\right)=M_{k}$ by part $(\mathrm{d})$ of the main induction hypothesis. Since $P$ is locally stratified and the level of $B_{i} \leq k$, $B_{i} \notin T_{P_{k+1}} \uparrow(n-1)\left(M_{k}\right)$.

By 1,2 and 3 above, $T_{P_{k+1}} \uparrow(n-1)\left(M_{k}\right) \models L_{1} \wedge \ldots \wedge L_{q}$. There is a clause $A \leftarrow L_{1}, \ldots, L_{q} \in$ $\operatorname{ground}(P)$. Hence, $A \in T_{P_{k+1}} \uparrow n\left(M_{k}\right)$.

(part (b)) We assume that $A \in T_{P_{k+1}} \uparrow n\left(M_{k}\right)$ and $\gamma_{0}+\ldots+\gamma_{k}+n \leq d$. Suppose first $n=1$. Then, there exists a clause $A \leftarrow L_{1}, \ldots, L_{q} \in \operatorname{ground}(P)(q \geq 0)$ such that $M_{k} \models L_{1} \wedge \ldots \wedge L_{q}$. There are two possibilities for each $L_{i}$.

1. If $L_{i}$ is a positive literal $B_{i} \in H_{j}(j \leq k)$ then $B_{i} \in M_{k}=T_{P_{k}} \uparrow \gamma_{k}\left(M_{k-1}\right)$ and $\gamma_{0}+\ldots+\gamma_{k} \leq d$. By part (b) of the main induction hypothesis, there is a successful depth-bounded BFNF-derivation sequence of length $\leq \gamma_{0}+\ldots+\gamma_{k}$ for $P \cup\left\{\leftarrow B_{i}\right\}$.

2. If $L_{i}$ is a negative literal $\neg B_{i}\left(B_{i} \in B_{P}\right)$, then $B_{i} \notin T_{P_{k+1}} \uparrow(n-1)\left(M_{k}\right)$. Since $P$ is locally stratified, $B_{i} \in H_{j}$ for some $j \leq k$ and $B_{i} \notin M_{k}$. By part (a) of the main induction hypothesis, if for some $n \leq d$ there is a successful depth-bounded BFNFderivation sequence of length $\leq n$ for $P \cup\left\{\leftarrow B_{i}\right\}$ then $B_{i} \in T_{P_{k}} \uparrow n\left(M_{k-1}\right)$. Since $B_{i} \notin T_{P_{k}} \uparrow n\left(M_{k-1}\right)$ for any $n<\omega$, for any $l \leq d$ there is no successful depth-bounded 
BFNF-derivation sequence of length $\leq l$. Then every depth-bounded BFNF-derivation sequence for $P \cup\left\{\leftarrow B_{i}\right\}$ is failed by Lemma 23 .

Combining these 1 and 2, there is a successful depth-bounded BFNF-derivation sequence of length $\leq \gamma_{0}+\ldots+\gamma_{k}$ for $P \cup\left\{\leftarrow L_{1}, \ldots, L_{q}\right\}$. Since this derivation sequence is ground, there is a successful depth-bounded BFNF-derivation sequence of length $\leq \gamma_{0}+\ldots+\gamma_{k}+1$ for $P \cup\{\leftarrow A\}$

Suppose next $1<n \leq d$. Then there exists a clause $A \leftarrow L_{1}, \ldots, L_{q} \in \operatorname{ground}(P)(q \geq$ $0)$ such that $T_{P_{k+1}} \uparrow(n-1)\left(M_{k}\right) \models L_{1} \wedge \ldots \wedge L_{q}$. There are three possibilities for each $L_{i}$.

1. If $L_{i}$ is a positive literal $B_{i} \in H_{j}$ for some $j \leq k$ then $B_{i} \in M_{k}=T_{P_{k}} \uparrow \gamma_{k}\left(M_{k-1}\right)$ and $\gamma_{0}+\ldots+\gamma_{k} \leq d$. By part (b) of the main induction hypothesis, there is a successful depth-bounded BFNF-derivation sequence of length $\leq \gamma_{0}+\ldots+\gamma_{k}$ for $P \cup\left\{\leftarrow B_{i}\right\}$.

2. If $L_{i}$ is a positive literal $B_{i} \in H_{k+1}$ then $B_{i} \in T_{P_{k+1}} \uparrow(n-1)\left(M_{k}\right)$ and $\gamma_{0}+\ldots+\gamma_{k}+$ $(n-1) \leq d$. By part (b) of the secondary induction hypothesis, there is a successful depth-bounded BFNF-derivation sequence of length $\leq \gamma_{0}+\ldots+\gamma_{k}+(n-1)$ for $P \cup\left\{\leftarrow B_{i}\right\}$.

3. If $L_{i}$ is a negative literal $\neg B_{i}\left(B_{i} \in B_{P}\right)$, then $B_{i} \notin T_{P_{k+1}} \uparrow(n-1)\left(M_{k}\right)$. Since $P$ is locally stratified, $B_{i} \in H_{j}$ for some $j \leq k$ and $B_{i} \notin M_{k}$. By part (a) of the main induction hypothesis, if for some $n \leq d$ there is a successful depth-bounded BFNFderivation sequence of length $\leq n$ for $P \cup\left\{\leftarrow B_{i}\right\}$ then $B_{i} \in T_{P_{k}} \uparrow n\left(M_{k-1}\right)$. Since $B_{i} \notin T_{P_{k}} \uparrow n\left(M_{k-1}\right)$ for any $n<\omega$, for any $l \leq d$ there is no successful depth-bounded BFNF-derivation sequence of length $\leq l$. Then every depth-bounded BFNF-derivation sequence for $P \cup\left\{\leftarrow B_{i}\right\}$ is failed by Lemma 23 .

Combining these 1, 2 and 3, there is a successful depth-bounded BFNF-derivation sequence of length $\leq \gamma_{0}+\ldots+\gamma_{k}+(n-1)$ for $P \cup\left\{\leftarrow L_{1}, \ldots, L_{q}\right\}$. Since this derivation sequence is ground, there is a successful depth-bounded BFNF-derivation sequence of length $\leq \gamma_{0}+\ldots+\gamma_{k}+n$ for $P \cup\{\leftarrow A\}$

(part (c)) We assume that $A \in T_{P_{k+1}} \uparrow n\left(M_{k}\right)$ and $d<\gamma_{0}+\ldots+\gamma_{k}+n$. Since $P$ is a weakly reducing program, $P$ is locally finite stratified by Theorem 22 . By Theorem 6 , $A \in T_{P_{k+1}} \uparrow \gamma_{k+1}\left(M_{k}\right)$ and

$$
\gamma_{0}+\ldots+\gamma_{k}+\gamma_{k+1} \leq \sharp\left(H^{k+1}\right) .
$$

Since $\sharp\left(H^{k+1}\right) \leq d$,

$$
\gamma_{0}+\ldots+\gamma_{k}+\gamma_{k+1} \leq d
$$

By part (b) of this theorem, there is a successful depth-bounded BFNF-derivation sequence of length $\leq \gamma_{0}+\ldots+\gamma_{k}+\gamma_{k+1} \leq d$. Hence, part (c) is proved.

Now we show the completeness of the depth-bounded BFNF-derivation procedure. 
Corollary 25. Let $P$ be a weakly reducing program containing function symbols, $L$ be a ground literal, and $d$ be a depth-bound. If $d \geq \sharp\left(\left.B_{P}\right|_{|L|}\right)$ then a depth-bounded BFNFderivation for $P \cup\{\leftarrow L\}$ is complete with respect to the perfect model semantics.

By Lemma 21, the depth-bound $\sharp\left(\left.B_{P}\right|_{|L|}\right)$ is computable. Thus, the perfect model $M_{P}$ for a weakly reducing program containing function symbols is computed by the depth-bounded BFNF-derivation.

Example 6. The program $\mathrm{P} 4$ is a weakly reducing program containing function symbols:

$$
\mathrm{P} 4=\left\{\begin{array}{l}
\operatorname{even}(s(x)) \leftarrow \neg \operatorname{even}(x) \\
\operatorname{even}(x) \leftarrow \operatorname{even}(x) \\
\operatorname{even}(0)
\end{array}\right\}
$$

Its Herbrand base $B_{\mathrm{P} 4}=\left\{\operatorname{even}\left(s^{\alpha}(0)\right) \mid \alpha<\omega\right\}$, and

$$
\left.B_{\mathrm{P} 4}\right|_{n}= \begin{cases}\phi & (n<2) \\ \left\{\operatorname{even}\left(s^{j}(0)\right) \mid j \leq n-2\right\} & (n \geq 2) .\end{cases}
$$

Thus, $\sharp\left(\left.B_{\mathrm{P} 4}\right|_{n}\right)$ is at most $n-1$. An atom even $(s(s(0)))$ is contained in the perfect model of $\mathrm{P} 4$, and $|\operatorname{even}(s(s(0)))|=4$. Hence, $\mathrm{P} 4 \cup\{\leftarrow \operatorname{even}(s(s(0)))\}$ has a successful depth-bounded BFNF-derivation with the depth-bound $3\left(=\sharp\left(\left.B_{\mathrm{P} 4}\right|_{4}\right)=4-1\right)$.

\section{Acknowledgements}

The author is grateful to Setsuo Arikawa for his comments and encouragement, and to Takeshi Shinohara and Akihiro Yamamoto for helpful discussions and comments.

\section{References}

[1] Apt, K.R. , Blair, H.A. , and Walker, A. , Towards a theory of declarative knowledge, in Minker, J. (ed.), Foundations of Deductive Databases and Logic Programming, Morgan Kaufmann, 1988.

[2] Arikawa, S. , Shinohara, T. , and Yamamoto, A. , Elementary formal system as a unifying framework for language learning, in Proc. of the Second Annual Workshop on Computational Learning Theory, Morgan Kaufmann, 1989.

[3] Arimura, H. , Completeness of depth-bounded resolution in logic programming, in 6th Conference Proceedings of Japan Society for Software Science and Technology, 1989.

[4] Cavedon, L. , Continuity, consistency, and completeness properties for logic programs, in Proc. of the Sixth International Conference on Logic Programming, 1989. 
[5] Fitting, M. , A Kripke-Kleene semantics for logic programs. J.Logic Programming 2(4) (1985).

[6] Jaffer, F. , Lassez, J. , and Maher, M. , A theory of complete logic programs with equality. J.Logic Programming 1(3) (1984).

[7] Kemp, D.B. and Topor, R.W. , Completeness of a top-down query evaluation procedure for stratified databases, in Proc. of the Fifth International Conference on Logic Programming, 1988.

[8] Knuth, D.E. , The Art of Computer Programming, volume 1, chapter 2. Addison Wesley, second edition, 1973.

[9] Lloyd, J.W. , Foundations of Logic Programming, Springer Verlag, second edition, 1987.

[10] Lloyd, J.W. and Topor, R.W. , A bases for deductive database systems II. J.Logic Programming 3(1) (1986).

[11] Maher, M.J. , Equivalence of logic programs, in Minker, J. (ed.), Foundations of Deductive Databases and Logic Programming, Morgan Kaufmann, 1988.

[12] Przymusinski, T. , On the declarative semantics of deductive databases and logic programs, in Minker, J. (ed.), Foundations of Deductive Databases and Logic Programming, Morgan Kaufmann, 1988.

[13] Reiter, R. , On closed world data bases, in Gallaire, H. and Minker, J. (eds.), Logic and Data Bases, Plenum, 1978.

[14] Seki, H. and Itoh, H. , A query evaluation method for stratified programs under the extended CWA, in Proc. of the Fifth International Conference on Logic Programming, 1988.

[15] Tärnlund, S.A., Horn clause computability. BIT 17(2) (1977).

[16] Van Gelder, A. , Negation as failure using tight derivations for genaral logic programs, in Minker, J. (ed.), Foundations of Deductive Databases and Logic Programming, Morgan Kaufmann, 1988.

[17] Yamamoto, A. , Elementary formal system as a logic programming language, in Proc. of the Logic Programming Conference '89, 1989. 


\section{About the Author}

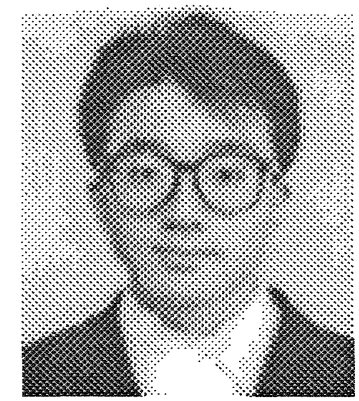

Hiroki Arimura (有村博紀) was born in Fukuoka on June 7, 1965. He received the B.S. degree from Kyusyu University in 1988. Presently, he is a graduate student of Master Course in Information Systems, Kyushu University. His interests are in logic programming and deductive database. 